Nanoparti cl e synt hesi s usi ng hi gh- power ed pul se- modul at ed i nduct i on ther mal pl asma

\begin{tabular}{|l|l|}
\hline 著者 & $\begin{array}{l}\text { Tanaka Yasunor i, Nagumo Takeo, Sakai H., } \\
\text { Uesugi Yoshi hi ko, Sakai Y., Nakamur a K. }\end{array}$ \\
\hline $\begin{array}{l}\text { j our nal or } \\
\text { publ i cat i on ti tl e }\end{array}$ & Journal of Physi cs D: Appl i ed Physi cs \\
\hline vol une & 43 \\
\hline number & 26 \\
\hline page range & 265201 \\
\hline year & $2010-01-01$ \\
\hline URL & ht t p: //hdl . handl e. net /2297/24811 \\
\hline
\end{tabular}




\title{
Nanoparticle synthesis using high-powered pulse-modulated induction thermal plasma
}

\author{
Yasunori Tanaka $\nmid$ T Nagumo, H Sakai, Y Uesugi, Y Sakai $\ddagger$, \\ K Nakamuraf \\ Faculty of Electrical Engineering and Computer Science, Kanazawa University, \\ Kakuma, Kanazawa 920-1192, JAPAN \\ $\ddagger$ Research Center for Production \& Technology, Nisshin Seifun Group Inc.,5-3-1 \\ Tsurugaoka, Fujimino 356-8511, JAPAN \\ E-mail: tanaka@ec.t.kanazawa-u.ac.jp
}

\begin{abstract}
Nanoparticle synthesis was performed using high-powered pulsemodulated induction thermal plasma (PMITP) technique to study the effect of coil current modulation on synthesized nanoparticles. This is the first paper to present a summary of results of $\mathrm{TiO}_{2}$ nanoparticle synthesis using high-power Ar- $\mathrm{O}_{2}$ PMITP at $20 \mathrm{~kW}$. The synthesized particles were analyzed using a field emission scanning electron microscope (FE-SEM), and X-ray diffractometry (XRD). In addition, optical emission spectroscopy (OES) was used during nanoparticle synthesis experiments to measure $\mathrm{TiO}$ spectra and to determine the time-averaged vibrational and rotational temperatures of $\mathrm{TiO}$ in the reaction chamber. Results showed that the PMITP produced smaller nanoparticles and a narrower size distribution of particles. Moreover, PMITP provided a lower-temperature region in the reaction chamber downstream of the plasma torch than such regions in non-modulated thermal plasmas.
\end{abstract}

Submitted to: J. Phys. D: Appl. Phys.

$\nmid$ To whom correspondence should be addressed (tanaka@ec.t.kanazawa-u.ac.jp) 


\section{Introduction}

High-pressure and high-power inductively coupled thermal plasmas (ICTPs) have become effective heat and chemical species sources for various material processes such as syntheses of diamond films [1,2], thermal barrier coatings [3, 4], syntheses of fullerene $[5,6]$, and materials surface modifications [7]. This has occurred because ICTPs can provide advantages of remarkably higher enthalpy and higher radical density than cold plasmas. They also cause little contamination because they use no electrodes. Such ICTP devices are also useful to synthesize nanoparticles of various kinds [8]-[15]. Titanium dioxide $\left(\mathrm{TiO}_{2}\right)$ nanoparticles are continually receiving attention for use as photocatalysts [16], photonic crystals [17], photovoltaic cells [18], and gas sensors [19]. They are also anticipated for use as strong deoxidation materials to produce hydrogen gas from water for fuel cells [20]. The $\mathrm{TiO}_{2}$ nanoparticle properties depend on the particle size, crystal structure, phase constituents, etc. Therefore, particle size and phase constituent control are extremely important to obtain the specified performance of $\mathrm{TiO}_{2}$ nanoparticles. Several studies have examined synthesis of $\mathrm{TiO}_{2}$ nanoparticles using a steady-state type of ICTP and have investigated control of its particle size and phase constituent $[10,11,15]$.

We have developed a high-power pulse-modulated induction thermal plasmas (PMITP) system for use at a rated power of $30 \mathrm{~kW}$ and have investigated its fundamental features [21]-[24]. The PMITP system has a function of amplitude modulation of the coil current sustaining the induction thermal plasma into a rectangular waveform. Such a millisecond modulation in the coil current can markedly perturb the thermal plasma. It can then change the temperature and radical densities as well as the gas flow field in the thermal plasma in a time domain [23, 24]. Moreover, we newly developed a highpower arbitrary-waveform-modulated induction thermal plasma (AMITP) system at a rated power of $20 \mathrm{~kW}$ for additional enhancement of the degrees of freedom in thermal plasma control [25]. The AMITP system can modulate the coil current not only into a rectangular waveform but also into an externally given waveform. It actually provides precise time-domain control of the temperature of thermal plasmas [25].

To date, applications of such high-power modulated induction thermal plasmas are being investigated for use in some material processing fields. For example, Ishigaki et al. applied Ar PMITP for surface modification of a $\mathrm{TiO}_{2}$ bulk specimen in a fundamental study [26]. Another application of PMITP is the work of Ohashi et al., who applied $\mathrm{Ar}-\mathrm{H}_{2}$ PMITP for hydrogen doping to $\mathrm{ZnO}$. Their results show that $\mathrm{H}$ atoms can be implanted into $\mathrm{ZnO}$ through treatment with an $\mathrm{Ar}-\mathrm{H}_{2}$ PMITP, thereby improving its photoluminescence $[27,28,29]$. The authors have also investigated application of the Ar- $\mathrm{N}_{2}$ PMITP for surface nitridation processing of materials [30]-[34]. Results of this work show that rectangular modulation of the coil current can provide an increased number of excited $\mathrm{N}$ atoms: simultaneously, it can provide decreased enthalpy flow onto a specimen irradiated by $\mathrm{Ar}-\mathrm{N}_{2}$ PMITP. This result implies high-speed nitridation of the specimen surface with less thermal damage. However, few reports in the relevant 
literature describe application of a high-power modulated induction thermal plasma to synthesis of nanoparticles, which are given great attention, as described above. In particular, the influence of coil current modulation on the order of several hundreds of amperes is rarely studied in nanoparticle synthesis.

This paper describes experimental results of $\mathrm{TiO}_{2}$ nanoparticle synthesis using Ar$\mathrm{O}_{2}$ PMITP at $20 \mathrm{~kW}$. This is the first paper to report systematic investigation results of nanoparticle synthesis using a high-powered PMITP. Mainly two effects can be expected in nanoparticle syntheses using coil current modulation: rapid and complete evaporation of injected raw materials during high-power input time duration, and rapid cooling of evaporated material during the low-power input period; and enhancement of timeaveraged temperature-gradient, which also causes cooling of evaporated material during transferral to the downstream chamber. Enhancement of the time-averaged temperature gradient has been seen in other applications of PMITP by experiments and numerical simulations in our previous works $[30,31,35]$. Many other papers have studied the effect of quenching gas flow into thermal plasmas on particle size, indicating that high quenching rate in thermal plasmas decreased particle size of nanoparticles [14, 15]. In this case of introduction of quenching gas flow, it is pointed out that higher temperature gradient in the quenching region of thermal plasmas is important to synthesize small size nanoparticles. The PMITP has a possibility to have higher quenching rate and higher temperature gradient in thermal plasmas, and to cause controllability of particle size by controlling the coil current modulation.

Our work described in this paper is also intended to clarify the effect of coil current modulation or disturbance in high-power thermal plasmas during nanoparticle synthesis. The synthesized particles were collected in two series reaction chambers and a downstream filter; then they were analyzed using FE-SEM for morphology and analyzed using XRD for surface compounds and phase identification of the synthesized nanoparticles. In addition, spectroscopic observations were conducted to measure emission spectra to investigate the gas state in the reaction chamber during nanoparticle synthesis. As a result, the temperature in the reaction chamber downstream of the plasma torch was found to be lower in the PMITP compared to that in non-modulated ICTP.

As the main contribution of this paper, the influences of the modulation degree in the coil current sustaining PMITP are summarized, especially its effects on the following: (i) the size distribution, the mean diameter and the standard deviation of the synthesized nanoparticles; (ii) the weight fraction of anatase $\mathrm{TiO}_{2}$ in the synthesized nanoparticles; and (iii) the temperature of plasma or gas in the reaction chamber in which nanoparticles are produced. We also emphasize this work's importance from the viewpoint of effects of disturbance in thermal plasmas on the characteristics of synthesized nanoparticles for effective efficiency of the nanoparticle synthesis method using thermal plasmas. This contribution will also be useful to elucidate the disturbance effects on synthesized nanoparticle during non-modulated plasma processing. 


\section{Experimental}

\subsection{Experimental arrangements}

Figure 1 portrays the experimental arrangements used for this study. The plasma torch is configured identically to that used in our previous work. Its details were described in an earlier paper $[23,24]$. The plasma torch has two coaxial quartz tubes. The interior quartz tube has $70 \mathrm{~mm}$ inner diameter; its length is $370 \mathrm{~mm}$. An argon-oxygen gas mixture was supplied as a sheath gas along the inside wall of the interior quartz tube from the top of the plasma torch. Titanium raw material was fed using a powder feeder with Ar carrier gas through a water-cooled tube probe. The water-cooled tube probe was inserted from the top of the plasma torch head, as depicted in figure 1. No other quenching gas was injected into the torch or chamber in this study. Downstream of the plasma torch, the water-cooled chambers were installed vertically and then horizontally, as depicted in figure 1. The total length of the vertical chamber is $600 \mathrm{~mm}$; its inner diameter is $130 \mathrm{~mm}$. Similarly, the total length of the horizontal chamber is $600 \mathrm{~mm}$; its inner diameter is $130 \mathrm{~mm}$. Further downstream of the horizontal chamber, a powdercollecting filter and the collecting chamber are set up. A vacuum pump is set up further downstream.

We used a radio frequency ( $\mathrm{rf}$ ) power supply with a metal-oxide semiconductor field emission transistor (MOSFET). The MOSFET rf power supply has a high dc-rf conversion efficiency of greater than $90 \%$. It can also modulate electric current of the order of several hundreds of amperes for supplying the coil current. The amplitude of the coil current sustaining induction thermal plasmas was modulated into a rectangular waveform in this work. The modulated coil current has modulation parameters such as the shimmer current level (SCL), the on-time, and the off-time [23, 24]. Figure 2 presents a definition of the modulation parameters. The 'on-time' is defined as the time duration with higher current level (HCL); the 'off-time' is the time duration with lower current level (LCL). The shimmer current level is defined as a ratio of the lower current level (LCL) to the higher current level (HCL). In addition, the duty factor (DF) is set to a ratio of the on time to one modulation cycle: $\mathrm{DF}=$ 'on-time' /('on-time' +'offtime'). Condition $100 \%$ SCL or $100 \%$ DF corresponds to the non-modulation condition. Condition SCL $<100 \%$ and $\mathrm{DF}<100 \%$ provides a modulation condition. Lower SCL engenders a larger modulation. Synthesized particles were collected in the upstream chamber, the downstream chamber, and in the filter.

\subsection{Experimental conditions}

The total flow rate of $\mathrm{Ar}-\mathrm{O}_{2}$ sheath gas was fixed at 80.0 standard litres per minute $(\mathrm{slpm})\left(=1.33 \times 10^{-3} \mathrm{~m}^{3} \mathrm{~s}^{-1}\right)$. The oxygen gas admixture ratio to Ar was $10 \%$ in the sheath gas in the gas flow rate. The gas flow rate of Ar carrier gas was 2 slpm. Titanium powder with mean diameter of $45 \mu \mathrm{m}$ is fed as the raw material. Figure 3 shows a photograph of the raw material. The powder feed rate $g$ of the Ti raw material 
was fixed around $3.5 \mathrm{~g} \mathrm{~min}^{-1}$ to $4.0 \mathrm{~g} \mathrm{~min}^{-1}\left(=5.8 \times 10^{-5} \mathrm{~kg} \mathrm{~s}^{-1}\right.$ to $\left.6.7 \times 10^{-5} \mathrm{~kg} \mathrm{~s}^{-1}\right)$. The pressure inside the chamber was controlled. It was fixed at 300 Torr $(=40 \mathrm{kPa})$ with an automatic feedback control for pressure. The on-time and the off-time were fixed respectively at $12 \mathrm{~ms}$ and $3 \mathrm{~ms}$. The duty factor (DF) was fixed at $80 \%$. In this case, one modulation cycle is $15 \mathrm{~ms}$. The modulation cycle was fixed at $15 \mathrm{~ms}$ because of the following facts: We conducted numerical simulation of the gas flow and temperature fields, which revealed that the gas flow velocity is about $20-40 \mathrm{~m} / \mathrm{s}$. From that result, the staying time of the injected powders in the plasma torch was estimated as $10-20$ $\mathrm{ms}$, and the residence time of the reactant vapour was also estimated to be $10-20 \mathrm{~ms}$. Therefore, we chose $15 \mathrm{~ms}$ as a modulation cycle to expect sufficient evaporation of the injected powders in the high-temperature region in the plasma during the on-time, and successive rapid cooling of the plasma tail during the off-time.

Under the fixed conditions described above, the shimmer current level (SCL) was changed from $100 \%-65 \%$. Although the electric coil current is modulated in a rectangular waveform, the time-averaged input power to the MOSFET rf power supply over a modulation cycle was controlled to be fixed at $20 \mathrm{~kW}$ independent of SCL. For fixed time-averaged input power for modulation operation, i.e. at SCL $<100 \%$, the higher current level (HCL) was set to higher value than that at SCL $=100 \%$, whereas the lower current level (LCL) was controlled to a lower value than that at $\mathrm{SCL}=100 \%$. Consequently, the instantaneous input power was modulated, although the time-averaged input power over one modulation cycle was fixed at $20 \mathrm{~kW}$. For example, the instantaneous input power reaches about $23 \mathrm{~kW}$ for the on-time, although the instantaneous input power decreases to about $11 \mathrm{~kW}$ for the final stage in the offtime in case of $\mathrm{SCL}=70 \%$. Adoption of the time-averaged input power fixed enables us to compare experimental results under the same electric power cost condition. In the experiments, the time-averaged input power was measured using a three phase ac wattmeter at the input terminal to the rf power supply. Experiments were made twice under the same conditions to verify the result.

\section{Coil current modulation effects on synthesized particles}

\subsection{SEM images and particle diameter distribution}

Figure 4 presents examples of FE-SEM micrographs of particles synthesized using induction thermal plasma with and without the coil current modulation. The panel $(a)$ portrays the image for $\mathrm{SCL}=100 \%$, i.e. non-modulation condition, whereas the panel (b) contains those for modulation conditions. In the panel (b), photographs (i) and (ii) respectively show images for $\mathrm{SCL}=90 \%$ and $\mathrm{SCL}=70 \%$. As shown there, smaller spherical nanoparticles were obtained under lower SCL conditions, especially at the lowest SCL condition at SCL $=70 \%$ in this case.

Next, the particle size distributions of synthesized particles were estimated. The particle size distributions were evaluated by measuring diameters of randomly selected 
200 particles in each of the SEM micrograph images. Figure 5 portrays the size distribution of particles synthesized using PMITP at different SCLs. Results show that ICTP with coil current modulation produces particles with smaller mean diameter than those produced using ICTP without coil current modulation. For example, the mean diameter $d_{\mathrm{m}}$ is $50.3 \mathrm{~nm}$ and the standard deviation $\sigma$ is $25.3 \mathrm{~nm}$ in the PMITP case of SCL $=70 \%$, where $d_{\mathrm{m}}$ is $63.2 \mathrm{~nm}$ and $\sigma$ is $30.6 \mathrm{~nm}$ for the induction thermal plasma without modulation. In other words, if the PMITP is used instead of a conventional induction thermal plasma without modulation, then we can obtain smaller nanoparticles.

Figure 6 shows the dependence of the mean diameter of synthesized particles on the shimmer current level (SCL). The error bars in this figure indicate fluctuations in the measured mean diameter of synthesized particles at different collecting positions. The SCL in the horizontal axis is decreased from the left-hand-side to the right-hand-side in this figure. It is readily apparent that reducing SCL from $80 \%$ to $65 \%$ decreases the mean particle diameter. Consequently, a larger modulation provides particles with a smaller mean diameter. Figure 7 depicts the dependence of the standard deviation in the diameter of synthesized particles on SCL. As shown in the figure, the standard deviation in the diameter has no strong dependence on SCL, although the modulation in the coil current had been expected to increase the standard deviation in the diameter of the synthesized particles.

To cross-check this dependence of the mean diameter on SCL, the specific surface area per unit mass was further measured using the Brunauer-Emmett-Teller (BET) method. The specific surface area is generally increased by decreasing particle diameter, and is used as an indicator of the particle diameter. Figure 8 presents the specific surface area of synthesized particles as a function of SCL. The specific surface area is roughly increased concomitantly with decreasing SCL from $80 \%$ to $65 \%$. This dependence of the specific surface area on the SCL agrees with that of estimated mean particle diameter on the SCL in figure 6.

As seen in figures 6 and 8, the mean particle diameter and the specific surface area little change with SCL between $100 \%$ and $80 \%$. These little changes in both figures mean that small modulation in the coil current scarcely affects or rather slightly increase the mean particle diameter. The possible reason why the mean particle diameter is increased from $\mathrm{SCL}=100 \%$ to $80 \%$ is inferred as follows: In our experimental condition, the timeaveraged input power was fixed irrespective of SCL. For this condition, the HCL in modulation cases is set to be higher than the current level at SCL $=100 \%$, and the LCL in modulation cases is lower than the current level at SCL $=100 \%$. In a on-time duration, a higher joule heating power is inputted to the plasma region, and thus the instantaneous plasma temperature in the torch increases with time to reach a higher value than that at $\mathrm{SCL}=100 \%$. To the contrary, the joule heating power decreases with time in the off-time duration, and the instantaneous plasma temperature in the torch decreases to reach a lower value than that at $\mathrm{SCL}=100 \%$. However, the time-averaged plasma temperature in the torch is considered to hardly change with SCL. On the other hand, in the reaction chamber downstream of the plasma torch where the joule heating 
power is very low, the instantaneous plasma temperature little changes with time for small modulation between $\mathrm{SCL}=100 \%$ and $\mathrm{SCL}=80 \%$. This is attributed to the fact that the plasma temperature in the reaction chamber is influenced only through thermal conduction and convection from the upstream plasma torch. From the above reason, the rapid cooling in the reaction chamber may hardly occur under such small modulation conditions. Furthermore, the mean diameter may increase depending on the powder injection timing against the modulation cycle. For large modulation conditions with SCL $\leq 70 \%$, the instantaneous temperature in the reaction chamber can largely change with time, and in addition the time-averaged gas temperature decreases with reducing SCL from $80 \%$ to $65 \%$ as described in later section. Therefore, reducing SCL to $65 \%$ is considered to provide the smaller nanoparticles.

\subsection{XRD spectra and fraction of anatase}

An X-ray diffraction (XRD) analysis was made to study the crystallographic structure and chemical composition of the synthesized nanoparticles. Figure 9 portrays examples of XRD spectra for particles synthesized using the ICTP without modulation and the PMITP. The designation 'A' in this figure indicates that the specified XRD spectral line arises from $\mathrm{TiO}_{2}$ in anatase form; the designation ' $\mathrm{R}$ ' denotes the lines originating from the $\mathrm{TiO}_{2}$ in rutile form. Almost identical XRD spectra were found for all particles synthesized using PMITP with different SCLs and ICTP without modulation. These spectral lines obtained in this analysis are all related to $\mathrm{TiO}_{2}$. No other spectral line exists, for example, from Ti raw materials. Therefore, the use of PMITP can provide $\mathrm{TiO}_{2}$ nanoparticles. They have almost identical ratios of anatase to rutile phases to $\mathrm{TiO}_{2}$ nanoparticles provided by the non-modulated induction thermal plasma. The weight fraction $f_{\mathrm{A}}$ of $\mathrm{TiO}_{2}$ in anatase form to $\mathrm{TiO}_{2}$ in rutile form can be estimated from the following equation $[10,15]$ :

$$
f_{\mathrm{A}}=\frac{1}{1+1.26 \frac{I_{\mathrm{R}_{27.4^{\circ}}}}{I_{\mathrm{A}_{25.3^{\circ}}}}},
$$

where $I_{\mathrm{R}_{27.4^{\circ}}}$ and $I_{\mathrm{A}_{25.3^{\circ}}}$ respectively signify the XRD spectral intensities at $2 \theta=27.4^{\circ}$ for rutile and $2 \theta=25.3^{\circ}$ for anatase. This equation was obtained using the test line for standard $\mathrm{TiO}_{2}$. It is pointed out that nucleation of anatase $\mathrm{TiO}_{2}$ tends to occur by higher degree of undercooling of molten materials according to the theoretical calculation of the critical free energy for nucleation of rutile and anatase $\mathrm{TiO}_{2}$ in a literature [36]. Furthermore, the ratio of anatase to rutile in $\mathrm{TiO}_{2}$ nanoparticles can be controlled by an admixture ratio of $\mathrm{O}_{2}$ to Ar from experimental results $[10,15]$.

Figure 10 presents the weight fraction of anatase $\mathrm{TiO}_{2}$ to rutile $\mathrm{TiO}_{2}$ as a function of SCL. As shown in that figure, even without the modulation of the coil current, the weight fraction of anatase $\mathrm{TiO}_{2}$ is 0.9 which is extremely high. This fraction value is similar to those from other reports $[10,15]$. This may be attributed to the high temperature gradient at the tail of the thermal plasmas, which provides high temperature history for evaporated materials there. In case of modulation in our experiments, the weight 
fraction of anatase $\mathrm{TiO}_{2}$ also remains 0.8-0.9, generally almost independent of SCL. This means that the PMITP keeps to provide enough higher temperature-quenching at the plasma tail region for nucleation of anatase $\mathrm{TiO}_{2}$.

Strictly speaking, there is a slight local minimum in the weight fraction of anatase $\mathrm{TiO}_{2}$ at $\mathrm{SCL}=80 \%$. This presence of the local minimum on SCL also indicates that cooling effect of molten materials are decreased around $\mathrm{SCL}=80 \%$, because the nucleation of anatase $\mathrm{TiO}_{2}$ can occur by higher degree of undercooling of molten materials as described above. The decreased cooling effect for the small modulation with SCL $>80 \%$ may be due to the similar reason why the mean particle diameter slightly increases with reducing SCL from $100 \%$ to $80 \%$ as discussed in the previous section. A further study is necessary to elucidate this dependence on SCL in future work.

\subsection{Spectroscopic observation during nanoparticle synthesis}

3.3.1. Observation of time-averaged emission spectra We mainly consider the two following effects in nanoparticle syntheses in a PMITP: The first effect is rapid evaporation of the injected raw material during the on-time and rapid cooling of it during off-time. This temperature modulation effect occurs markedly in the plasma torch in an interval of milliseconds during synthesis of nanoparticles. It also occurs downstream of the plasma torch for large modulation condition. The second effect is the enhancement of time-averaged temperature gradient, which also causes cooling of evaporated material during its transferral to the downstream chamber. As described in this paper, the second effect is investigated for the first approach.

Optical emission spectroscopy (OES) measurement was conducted from the viewwindow installed on the reaction chamber during synthesis of nanoparticles. Based on those measurements, we investigated the thermal plasma state in the reaction chamber downstream of the plasma torch where nanoparticles are formed. Figure 11 depicts the spectroscopic observation system and three observation positions: at $200 \mathrm{~mm}$ below the coil end, at $370 \mathrm{~mm}$ below the coil end, and at $460 \mathrm{~mm}$ below the coil end. The observation region of the focal plane for each of observation positions was about $8 \mathrm{~mm}$ in diameter. The light from the observation position was transmitted through an optical fibre bundle to the slit of a polychromator with an ICCD element. The combination of the polychromator and the ICCD can measure the light intensity in a wavelength range of 300-1200 $\mathrm{nm}$ with a wavelength resolution of $1 \mathrm{~nm}$. The device function of this system was measured; it had a full width at half maximum of $2 \mathrm{~nm}$. The exposure time of the ICCD used here was set to $30 \mathrm{~ms}$. This time scale of $30 \mathrm{~ms}$ is sufficiently long to include two modulation cycles. The adoption of this long exposure time allows one to measure the time-accumulated radiation intensity from the plasma. In addition, such an observation was conducted 30 times, and the averaged radiation intensities from emission spectra were computed to enhance the signal-to-noise $(\mathrm{S} / \mathrm{N})$ ratio. It is noted that measured radiation intensity is the integrated radiation intensity along the line of sight, and that no Able inversion was applied. Thus, the measured radiation intensity 
is the integrated and time-averaged radiation intensity, which can be useful to obtain rough tendency of the spectral radiation intensity for comparison between modulation and no modulation cases. The sensitivity of the whole optical system including the lens, the optical fiber bundle, the polychromator and the ICCD was calibrated for different wavelengths using a standard light source with a tungsten-halide lamp.

Figure 12 shows the observed emission spectra at wavelengths of 400-850 nm. Panel $(a)$ in that figure shows spectra measured at $200 \mathrm{~mm}$ below the coil end; panels $(b)$ and (c) respectively show the spectra measured at $370 \mathrm{~mm}$ and $460 \mathrm{~mm}$ below the coil end. Each of panels has spectra at different SCL of 100\%, 70\% and 65\%. Remember that the radiation intensities of observed spectra here are all integrated along the line of sight at the observation position. For all cases, $\mathrm{TiO}$ molecular spectra as well as Ar, O and Ti atomic spectral lines were observed [37]. The radiation intensity of $\mathrm{TiO}$ spectra decreases as the observation position was set downstream from $200 \mathrm{~mm}$ to 460 $\mathrm{mm}$ below the coil end. This decrease in the radiation intensity seems to arise as the plasma/gas temperature and $\mathrm{TiO}$ density in the water-cooled chamber decrease to the downstream portion. On the other hand, the radiation intensity of TiO spectra strongly decreases concomitantly with decreasing SCL at any observation position. This might be attributed to the strong decrease in the plasma/gas temperature by modulation of the coil current, although the time-averaged input power was fixed at $20 \mathrm{~kW}$.

3.3.2. Calculation of emission coefficients of $\mathrm{TiO}$ The vibrational temperature $T_{\text {vib }}$ and the rotational temperature $T_{\text {rot }}$ of $\mathrm{TiO}$ molecules were estimated to confirm the decrease in the time-averaged temperature using the coil current modulation. The $T_{\text {vib }}$ and $T_{\text {rot }}$ can be determined by fitting the theoretically calculated radiation intensity of $\mathrm{TiO}$ spectra to that of the experimentally measured $\mathrm{TiO}$ spectra. The $\mathrm{TiO}$ spectra have three main transition systems of 'Red system $\left(A^{3} \Phi-X^{3} \Delta\right)$ ', 'Orange-Red system $\left(B^{3} \Pi-X^{3} \Delta\right)$ ', and 'Blue-Green system $\left(C^{3} \Delta-X^{3} \Delta\right)$ ' in the visible wavelength range of 400-800 $\mathrm{nm}$ [37]. The emission coefficient of $\mathrm{TiO}$ spectra for these systems was calculated theoretically on the assumption of a Boltzmann distribution for the population of excited particles. The emission coefficient $\varepsilon\left(\lambda_{n^{\prime} n^{\prime \prime} v^{\prime} v^{\prime \prime} J^{\prime} J^{\prime \prime}}\right)$ of the light emitted by transition from the upper excited level $\left(n^{\prime}, v^{\prime}, J^{\prime}\right)$ to the lower excited level $\left(n^{\prime \prime}, v^{\prime \prime}, J^{\prime \prime}\right)$ in $\mathrm{TiO}$ molecules is written as follows $[38,39]$ :

-Emission coefficient:

$$
\varepsilon\left(\lambda_{n^{\prime} n^{\prime \prime} v^{\prime} v^{\prime \prime} J^{\prime} J^{\prime \prime}}\right)=\frac{h c}{\lambda_{n^{\prime} n^{\prime \prime} v^{\prime} v^{\prime \prime} J^{\prime} J^{\prime \prime}}} N_{n^{\prime} v^{\prime} J^{\prime}} A_{n^{\prime} n^{\prime \prime} v^{\prime} v^{\prime \prime} J^{\prime} J^{\prime \prime}}
$$

-Number density of molecules excited at the upper level $\left(n^{\prime}, v^{\prime}, J^{\prime}\right)$ :

$$
\begin{gathered}
N_{n^{\prime} v^{\prime} J^{\prime}}=\frac{N\left(T_{\mathrm{ex}}, T_{\mathrm{vib}}, T_{\mathrm{rot}}\right)}{Z\left(T_{\mathrm{ex}}, T_{\mathrm{vib}}, T_{\mathrm{rot}}\right)}\left(2 J^{\prime}+1\right) \cdot \exp \left(-\frac{h c T_{e^{\prime}}}{k T_{\mathrm{ex}}}\right) \\
\cdot \exp \left(-\frac{h c F_{v^{\prime}}\left(J^{\prime}\right)}{k T_{\mathrm{rot}}}\right) \cdot \exp \left(-\frac{h c G_{e}\left(v^{\prime}\right)}{k T_{\mathrm{vib}}}\right)
\end{gathered}
$$

-Rotational energy:

$$
F_{v}(J)=B_{v} J(J+1)-D_{v} J^{2}(J+1)^{2}
$$




$$
\begin{aligned}
& B_{v}=B_{e}-\alpha_{e}\left(v+\frac{1}{2}\right) \\
& D_{v}=D_{e}+\beta_{e}\left(v+\frac{1}{2}\right)
\end{aligned}
$$

-Vibrational energy:

$$
G_{e}(v)=\omega_{e}\left(v+\frac{1}{2}\right)-\omega_{e} x_{e}\left(v+\frac{1}{2}\right)^{2}+\omega_{e} y_{e}\left(v+\frac{1}{2}\right)^{3}
$$

-Transition probability from the upper excited level $\left(n^{\prime}, v^{\prime}, J^{\prime}\right)$ to the lower excited level $\left(n^{\prime \prime}, v^{\prime \prime}, J^{\prime \prime}\right)$ :

$$
\begin{aligned}
& A_{n^{\prime} n^{\prime \prime} v^{\prime} v^{\prime \prime} J^{\prime} J^{\prime \prime}}=A_{n^{\prime} n^{\prime \prime}} q_{v^{\prime} v^{\prime \prime}} \frac{S_{J^{\prime}}^{\mathrm{P}, \mathrm{Q}, \mathrm{R}}}{2 J^{\prime}+1} \\
& A_{n^{\prime} n^{\prime \prime}}=\frac{64 \pi^{4} e^{2}}{3 h c^{3} \lambda_{n^{\prime} n^{\prime \prime} v^{\prime} v^{\prime \prime} J^{\prime} J^{\prime \prime}}^{3}}\left|R_{e}^{n^{\prime} n^{\prime \prime}}\right|^{2}
\end{aligned}
$$

-Line strengths by Hönl-London formulae for P, Q, and R branches:

(i) for $A^{3} \Phi-X^{3} \Delta$ transition:

$$
\begin{aligned}
S_{J^{\prime}}^{\mathrm{P}} & =\frac{\left(J^{\prime}+1+\Lambda^{\prime}\right)\left(J^{\prime}+2+\Lambda^{\prime}\right)}{4\left(J^{\prime}+1\right)} \\
S_{J^{\prime}}^{\mathrm{Q}} & =\frac{\left(J^{\prime}-\Lambda^{\prime}\right)\left(J^{\prime}+1+\Lambda^{\prime}\right)\left(2 J^{\prime}+1\right)}{4 J^{\prime}\left(J^{\prime}+1\right)} \\
S_{J^{\prime}}^{\mathrm{R}} & =\frac{\left(J^{\prime}-\Lambda^{\prime}\right)\left(J^{\prime}-1-\Lambda^{\prime}\right)}{4 J^{\prime}}
\end{aligned}
$$

(ii) for $B^{3} \Pi-X^{3} \Delta$ transition:

$$
\begin{aligned}
S_{J^{\prime}}^{\mathrm{P}} & =\frac{\left(J^{\prime}+1-\Lambda^{\prime}\right)\left(J^{\prime}+2-\Lambda^{\prime}\right)}{4\left(J^{\prime}+1\right)} \\
S_{J^{\prime}}^{\mathrm{Q}} & =\frac{\left(J^{\prime}+\Lambda^{\prime}\right)\left(J^{\prime}+1-\Lambda^{\prime}\right)\left(2 J^{\prime}+1\right)}{4 J^{\prime}\left(J^{\prime}+1\right)} \\
S_{J^{\prime}}^{\mathrm{R}} & =\frac{\left(J^{\prime}+\Lambda^{\prime}\right)\left(J^{\prime}-1+\Lambda^{\prime}\right)}{4 J^{\prime}}
\end{aligned}
$$

(iii) for $C^{3} \Delta-X^{3} \Delta$ transition:

$$
\begin{aligned}
S_{J^{\prime}}^{\mathrm{P}} & =\frac{\left(J^{\prime}+1+\Lambda^{\prime}\right)\left(J^{\prime}+1-\Lambda^{\prime}\right)}{J^{\prime}+1} \\
S_{J^{\prime}}^{\mathrm{Q}} & =\frac{\Lambda^{\prime 2}\left(2 J^{\prime}+1\right)}{J^{\prime}\left(J^{\prime}+1\right)} \\
S_{J^{\prime}}^{\mathrm{R}} & =\frac{\left(J^{\prime}+\Lambda^{\prime}\right)\left(J^{\prime}-\Lambda^{\prime}\right)}{J^{\prime}}
\end{aligned}
$$

-Wavelength of the light emitted by transition from the upper excited level $\left(n^{\prime}, v^{\prime}, J^{\prime}\right)$ to the lower excited level $\left(n^{\prime \prime}, v^{\prime \prime}, J^{\prime \prime}\right)$ :

$$
\frac{1}{\lambda_{n^{\prime} n^{\prime \prime} v^{\prime} v^{\prime \prime} J^{\prime} J^{\prime \prime}}}=T_{e^{\prime}}-T_{e^{\prime \prime}}+G_{e}\left(v^{\prime}\right)-G_{e}\left(v^{\prime \prime}\right)+F_{v^{\prime}}\left(J^{\prime}\right)-F_{v^{\prime \prime}}\left(J^{\prime \prime}\right)
$$


where $T_{e}$ is the electronic energy in wavenumber, $N\left(T_{\mathrm{ex}}, T_{\mathrm{vib}}, T_{\mathrm{rot}}\right)$ is the total number density of the molecules, $Z\left(T_{\mathrm{ex}}, T_{\mathrm{vib}}, T_{\mathrm{rot}}\right)$ is the internal partition function of the molecules, $q_{v^{\prime} v^{\prime \prime}}$ is the Franck-Condon factor for the transition from $v^{\prime}$ to $v^{\prime \prime},\left|R_{e}^{n^{\prime} n^{\prime \prime}}\right|$ is the electronic moment for transition from $n^{\prime}$ to $n^{\prime \prime}, h$ is Planck's constant, $k$ is Boltzmann's constant, $c$ is the velocity of light, $T_{\mathrm{ex}}$ is the electronic excitation temperature, $T_{\mathrm{vib}}$ is the vibrational temperature, $T_{\text {rot }}$ is the rotational temperature, $B_{e}$ and $D_{e}$ are the rotational constants, $\omega_{e}, \omega_{e} x_{e}$ and $\omega_{e} y_{e}$ are the vibrational constants, and $\Lambda$ is the axial component of orbital angular momentum for electrons in molecules. The transition probability $A_{n^{\prime} n^{\prime \prime}}$ was estimated using the measured lifetime value of excited $\mathrm{TiO}$ molecules [40]. The Franck-Condon factor $q_{v^{\prime} v^{\prime \prime}}$ of $\mathrm{TiO}$ was found in reference [41]. The quantities $T_{e}$ $B_{e}, D_{e}, \alpha_{e}$, and $\beta_{e}$ were obtained from the literature [40].

The theoretically calculated emission coefficient was further convoluted using the device function $W(\lambda)$ of the spectroscopic observation system to produce the calculated radiation intensity $I_{\text {cal }}(\lambda)$ as

$$
I_{\text {cal }}(\lambda)=\int_{0}^{\infty} W(\xi-\lambda) \varepsilon(\xi) d \xi
$$

where $\lambda$ signifies the wavelength. The device function $W(\lambda)$ of the spectroscopic observation system used in this work was measured using a low-pressure mercury lamp. Its full width at half maximum was $2 \mathrm{~nm}$.

Figure 13 presents the calculated radiation intensity $I_{\text {cal }}(\lambda)$ of $\mathrm{TiO}$ in arbitrary units for $A^{3} \Phi-X^{3} \Delta, B^{3} \Pi-X^{3} \Delta$ and $C^{3} \Delta-X^{3} \Delta$ systems in visible wavelengths of 400-900 $\mathrm{nm}$ in the case of $T_{\mathrm{ex}}=T_{\mathrm{vib}}=T_{\text {rot }}=4000 \mathrm{~K}$. The $A-X$ transition emits higher intensity light than the others at wavelengths of $600-900 \mathrm{~nm}$, as depicted in figure 13(a). On the other hand, the $B-X$ transition emits light with higher intensity for 500-800 nm, whereas the $C-X$ transition does it for 400-700 nm. The total calculated radiation intensity was obtained as shown in figure $13(b)$, which shows TiO molecular for wavelengths of 400-900 nm.

\subsubsection{Estimation of vibrational and rotational temperatures of TiO Temperatures $T_{\mathrm{vib}}$} and $T_{\text {rot }}$ were determined by fitting the theoretically calculated radiation intensity of $\mathrm{TiO}$ spectra to that of the experimentally measured $\mathrm{TiO}$ spectra. Convoluting $W(\lambda)$ with theoretically calculated emission coefficient to produce $I_{\text {cal }}(\lambda)$ and fitting $I_{\text {cal }}(\lambda)$ enables temperature estimation with a certain validity inspite of low wavelength resolution of the system. Before the fitting process, the radiation intensity of continuum component was subtracted from the calculated and measured radiation intensity because the actual continuum component can include complex continuum spectra from recombination radiation and other polyatomic molecular spectra, etc. Figure 14 depicts a fitting aspect between the measured and calculated radiation intensities of $\mathrm{TiO}$ spectra. The calculated radiation intensity for $T_{\text {vib }}=4700 \mathrm{~K}$ and $T_{\text {rot }}=4000 \mathrm{~K}$ fairly agrees with the measured one in this case. Here again, it is noteworthy that the $T_{\text {vib }}$ and $T_{\text {rot }}$ thus determined is the time-averaged ones. 
Similarly, $T_{\text {vib }}$ and $T_{\text {rot }}$ were determined at different observation positions for different SCL. Figure 15(a) and (b) respectively present the time-averaged $T_{\text {vib }}$ and $T_{\text {rot }}$ versus a distance from the coil end in the PMITP and also in the non-modulated plasma. Error bars were also added in this figure, indicating a fluctuation in the determined temperatures. As figure 15(a) portrays, the time-averaged $T_{\text {vib }}$ are decreased by 1000 $2000 \mathrm{~K}$ using the coil current modulation at SCL $<100 \%$ compared to the condition of non-modulation, i.e. $\mathrm{SCL}=100 \%$. Similarly, the time-averaged $T_{\text {rot }}$ are decayed by $1000 \mathrm{~K}$ at $\mathrm{SCL}=70 \%$ and $65 \%$ from the $T_{\text {rot }}$ at $\mathrm{SCL}=100 \%$. These results imply that the coil current modulation decreases the electron and gas temperatures in the chamber. This is true because $T_{\text {vib }}$ tends to be affected by the electron temperature, whereas $T_{\text {rot }}$ tends to be affected by the gas temperature in these experimental conditions. However, we found in other experiments that the time-averaged Ar excitation temperature in a high-temperature region at $10 \mathrm{~mm}$ below the coil end in the plasma torch remains at about $10000 \mathrm{~K}$ almost independent of SCL, although instantaneous Ar excitation temperature was strongly modulated [23]. Consequently, a higher temperature-gradient is formed from the plasma torch to the chamber in a modulated plasma than in a non-modulated plasma, in terms of the time-averaged temperature distribution. Such a higher temperature gradient might prevent excessive growth of particles after the initial growth nanoparticles. The higher axial gradient of the temperature in a PMITP than in a non-modulated ICTP is attributed to the fact that the PMITP has a wider diameter but a shorter length of the time-averaged high-temperature region in the plasma torch, which was found through numerical simulation [35]. This wider diameter of PMITP increases a power loss to the cooling water in the torch.

3.3.4. Errors in the temperatures determined In our determination of $T_{\mathrm{vib}}$ and $T_{\text {rot }}$, there are some uncertainties and procedures to cause errors. First, the measured radiation intensity of $\mathrm{TiO}$ spectra is the intensity integrated along the line of sight. In the actual situation, there are expected a temperature gradient and also a TiO density gradient along the light of sight. Inspite of the above fact, we did not perform the Abel inversion to determine $T_{\text {vib }}$ and $T_{\text {rot }}$. This is because the plasma is not always axis-symmetric from the fact that Ti material powder injected in the plasma is not axissymmetrically distributed: There is also a fluctuation in the distribution of the injected powder and evaporated materials. Thus, the Abel inversion can engender a rather error and is thus not useful to obtain the actual distribution of the radiation intensity of the Ti spectra in our case.

The existing temperature gradient can cause an error in the temperature determination. We investigated errors in the determination of $T_{\text {vib }}$ and $T_{\text {rot }}$ if there is a temperature gradient with a uniform vapor concentration in the plasma torch along the line of sight using a numerical simulation technique. Various type of the temperature distribution were assumed, and the emission coefficients were calculated and then they were integrated along the light of sight. For the calculated and integrated emission coefficients, the same fitting procedure was adopted to determine $T_{\text {vib }}$ and $T_{\text {rot }}$. It was 
found that both apparent $T_{\mathrm{vib}}$ and $T_{\text {rot }}$ were determined roughly to the temperatures at the highest radiation intensity region along the light of sight. Thus, $T_{\text {vib }}$ and $T_{\text {rot }}$ obtained in our experiments were inferred to be the temperatures approximately at the highest radiation intensity region of $\mathrm{TiO}$ spectra. The difference between the determined temperature and the actual temperature depends on the temperature distribution. For a typical temperature distribution in the induction thermal plasma, the determined $T_{\text {vib }}$ and $T_{\text {rot }}$ were estimated to be 500-600 K lower than the actual temperatures from the numerical simulation.

In addition, the measured radiation intensity of $\mathrm{TiO}$ spectra is the time-integrated radiation intensity for $30 \mathrm{~ms}$ which was set intentionally to be longer than the modulation cycle. This is because the influence of a temporal fluctuation in the injected powder on the temperature determination would be reduced. This procedure also cause an error in the temperature determination. The $T_{\text {vib }}$ and $T_{\text {rot }}$ determined from the timeintegrated radiation intensity are rather approximate to the ones at the highest radiation intensity duration in a modulation cycle. The errors due to the time-integration were also estimated to cause about $500-700 \mathrm{~K}$ in some cases. On the other hand, the light absorption of molecules can affect the accuracy in the determined temperature [42]. In the present calculation, we did not take into account this effect.

From the above reasons, the determined temperatures are close to the temperatures at the highest radiation region along the line of sight at the highest timing in a modulation cycle, and they have errors about $\pm 500-800 \mathrm{~K}$ at least.

\section{Conclusions}

In summary, a $20 \mathrm{~kW}$ class of Ar- $\mathrm{O}_{2}$ pulse-modulated induction thermal plasma was used for synthesis of $\mathrm{TiO}_{2}$ nanoparticles. Results showed that the coil current modulation caused lower temperature in the chamber. Furthermore, smaller nanoparticles were obtained than were obtained in the non-modulation condition. The weight fraction of anatase $\mathrm{TiO}_{2}$ in the synthesized particles was estimated as $80 \%-90 \%$ almost independent of the modulation degree in the coil current. The present results imply that PMITP is a promising mean of controlling the physical characteristics of nanoparticles.

\section{References}

[1] Berghaus J O, Meunier J L, and Gitzhofer F 2004 Monitoring and control of RF thermal plasma diamond deposition via substrate biasing Meas. Sci. Technol. 15 161-4

[2] Matsumoto S, Hino M, and Kobayashi T 1987 Synthesis of diamond films in an rf induction thermal plasma Appl. Phys. Lett. 51 737-9

[3] Huang H, Eguchi K, and Yoshida T 2003 Novel structured yttria-stabilized zirconia coatings fabricated by hybrid thermal plasma spraying. Sci. \& Technol. Advanced Mater. 4 617-22

[4] Chen W R, Wu X, Marple B R, and Patnaik P C 2005 Oxidation and crack nucleation/growth in an air-plasma-sprayed thermal barrier coating with NiCrAlY bond coat. Surface 8 Coatings Technol. 197 109-15 
[5] Wang C, Inazaki A, Shirai T, Tanaka Y, Sakuta T, Takikawa H, and Matsuo H 2003 Effect of ambient gas and pressure on fullerene synthesis in induction thermal plasma. Thin Solid Films $42541-8$

[6] Todorovic-Markovic B, Markovic Z, Mohai I, Karoly Z, Gal L, Foglein K, Szabo P T, and Szepvolgyi J 2003 Efficient synthesis of fullerenes in RF thermal plasma reactor Chem. Phys. Lett. 378 434-9

[7] Tanaka H, Osawa T, Moriyoshi Y, Kurihara M, Maruyama S, and Ishigaki T 2004 Improvement of the anode performance of graphite particles through surface modification in RF thermal plasma Thin Solid Films 457 209-16

[8] Girshick S L, Chiu C P, Muno R, Wu C Y, Yang L, Singh S K, and McMurry P H 1993 Thermal plasma synthesis of ultrafine iron particles J. Aerosol Sci. 24 367-82

[9] Shigeta M, Watanabe T, and Nishiyama H 2004 Numerical investigation for nano-particle synthesis in an RF inductively coupled plasma. Thin Solid Films 457 192-200

[10] Oh S M and Ishigaki T 2004 Preparation of pure rutile and anatase $\mathrm{TiO}_{2}$ nanopowders using $\mathrm{RF}$ thermal plasma Thin Solid Films 457 186-91

[11] Lee J E, Oh S M, and Park D W 2004 Synthesis of nano-sized Al doped $\mathrm{TiO}_{2}$ powders using thermal plasma Thin Solid Films 457 230-4

[12] Ishigaki T, Oh S M, Li J G, and Park D W 2005 Controlling the synthesis of TaC nanopowders by injecting liquid precursor into RF induction plasma Sci. \& Technol. Advanced Mater. 6 111-8

[13] Tong L and Reddy R G 2006 Thermal plasma synthesis of SiC nano-powders/nano-fibers Materials Research Bulletin 41 2303-10

[14] Ishigaki T and Li J G 2007 Synthesis of functional nanocrystallites through reactive thermal plasma processing Sci. \& Technol. Advanced Mater. 8 617-23

[15] Li J G, Ikeda M, Ye R, Moriyoshi Y, and Ishigaki T 2007 Control of particle size and phase formation of $\mathrm{TiO}_{2}$ nanoparticles synthesized in RF induction plasma J. Phys. D: Appl. Phys. $402348-53$

[16] Malato S, Blanco J, Alarcon D C, Maldonado M I, Fernandez-Ibanez P, and Gernjak W 2007 Photocatalytic decontamination and disinfection of water with solar collectors Catalysis Today $122137-49$

[17] Li J, Zhao X, Wei H, Gu Z Z, and Lu Z 2008 Macroporous ordered titanium dioxide $\left(\mathrm{TiO}_{2}\right)$ inverse opal as a new label-free immunosensor. Analytica Chemica Acta 625 63-9

[18] Gratzel M 2001 Photoelectrochemical cells. Nature 414 338-44

[19] Biju K P and Jain M K 2008 Effect of crystallization on humidity sensing properties of sol-gel derived nanocrystalline $\mathrm{TiO}_{2}$ thin films. Thin Solid Films $\mathbf{5 1 6} 2175-80$

[20] Park J H, Park O O, and Kim S 2006 Photoelectrochemical water splitting at titanium dioxide nanotubes coated with tungsten trioxide. Appl. Phys. Lett. 89163106

[21] Ishigaki T, Xiaobao F, Sakuta T, Banjo T, and Shibuya Y 1997 Generation of pulse-modulated induction thermal plasma at atmospheric pressure. Appl. Phys. Lett. 71 3787-9

[22] Tanaka Y and Sakuta T 2000 Measurement of dynamic response time in pulse modulated thermal plasma. Trans. Mater. Res. Soc. Japan 25 293-6

[23] Tanaka Y and Sakuta T 2003 Temperature control of Ar induction thermal plasma with diatomic molecular gases by pulse-amplitude modulation of coil-current. Plasma Sources Sci. E Technol. $1269-77$

[24] Tanaka Y, Uesugi Y, and Sakuta T 2007 Controlling the number of excited atoms flowing into the reaction chamber using pulse-modulated induction thermal plasmas at atmospheric pressure Plasma Sources Sci. \& Technol. 16 281-9

[25] Tanaka Y, Morishita Y, Okunaga K, Fushie S, and Uesugi Y 2007 Generation of high-power arbitrary-waveform modulated inductively coupled plasmas for materials processing Appl. Phys. Lett. 90071502

[26] Ishigaki T, Haneda H, Okada N, and Ito S 2001 Surface modification of titanium oxide in pulsemodulated induction thermal plasma Thin Solid Films 390 20-5

[27] Ohashi N, Ishigaki T, Okada N, Sekiguchi T, Sakaguchi I, and Haneda H 2002 Effect of hydrogen 
doping on ultraviolet emission spectra of various types of ZnO. Appl. Phys. Lett. 80 2869-71

[28] Ohashi N, Ishigaki T, Okada N, Taguchi H, Sakaguchi I, Hishita S, Sekiguchi T, and Haneda H 2003 Passivation of active recombination centers in $\mathrm{ZnO}$ by hydrogen doping. J. Appl. Phys. 93 6386-92

[29] Ohashi N, Wang Y G, Ishigaki T, Wada Y, Taguchi H, Sakaguchi I, Ohgaki T, Adachi Y, and Haneda H 2007 Lowered stimulated emission threshold of zinc oxide by hydrogen doping with pulsed argon-hydrogen plasma. J. Crystal Growth 306 316-20

[30] Tanaka Y, Muroya T, Hayashi K, and Uesugi Y 2006 Simultaneous control of numerical enhancement of $\mathrm{N}$ atom and decrease in heat flux into reaction chamber using $\mathrm{Ar}-\mathrm{N}_{2}$ pulsemodulated induction thermal plasmas. Appl. Phys. Lett. 89031501

[31] Tanaka Y 2006 Time-dependent two-temperature chemically non-equilibrium modelling of highpower $\mathrm{Ar}-\mathrm{N}_{2}$ pulse-modulated inductively coupled plasmas at atmospheric pressure. J. Phys. D: Appl. Phys. 39 307-19

[32] Tanaka Y, Muroya T, Hayashi K, and Uesugi Y 2007 Control of nitrogen atomic density and enthalpy flow into reaction chamber in $\mathrm{Ar}-\mathrm{N}_{2}$ pulse-modulated induction thermal plasmas. IEEE Trans. Plasma Sci. 35 197-203

[33] Tanaka Y, Hayashi K, Nakamura T, and Uesugi Y 2008 Influence of ontime on increased number density of excited nitrogen atom in pulse-modulated induction thermal plasmas. J. Phys. D: Appl. Phys. 41185203

[34] Ito T, Tanaka Y, Hayashi K, Uesugi Y 2009 Dynamic behavior of hydrogen and nitrogen radicals in pulse modulated induction thermal plasmas J. Plasma \&3 Fusion Res. Ser. 8, 728-31

[35] Ye R, Murphy A B, and Ishigaki T 2007 Numerical modeling of an Ar- $\mathrm{H}_{2}$ radio-frequency plasma reactor under thermal and chemical nonequilibrium conditions. Plasma Chem. \& Plasma Process. 27 189-204

[36] Li Y and Ishigaki T 2002 Thermodynamic analysis of nucleation of anatase and rutile from $\mathrm{TiO}_{2}$ melt J. Cryst. Growth 242 511-516

[37] Pearse R W B and Gaydon A G 1976 The identification of molecular spectra 4-th ed. pp.347-9 Chapman \& Hall: New York

[38] Herzberg G 1950 Molecular spectra and molecular structure vol I. Spectra of diatomic molecules, Van Nostrand: New York.

[39] Herzberg G 1971 The spectra and structure of simple free radicals. An introduction to molecular spectroscopy. Cornell University Press.

[40] Herzberg G 1979 Molecular spectra and molecular structure vol IV. Constants of diatomic molecules, Litton: London.

[41] Bell R A, Dwivedi P H, Branch D, and Huffaker J N 1979 Rotational dependence of FranckCondon factors selected band systems of $\mathrm{TiO}, \mathrm{ZrO}, \mathrm{MgO}, \mathrm{LaO}, \mathrm{SiO}$. Astrophys. J. Suppl. Ser. $41593-630$

[42] Saidane K, Razafinimanana M, Lange H, Huczko A, Baltas M, Gleizes A, and Meunier J-L 2004 Fullerene synthesis in the graphite electrode arc process: local plasma characteristics and correlation with yield J. Phys. D: Appl. Phys. 37 232-239 


\section{Figure captions}

Figure 1. Experimental setup for nanoparticle synthesis.

Figure 2. Modulated coil current and definition of modulation parameters.

Figure 3. Image of titanium raw powder.

Figure 4. SEM micrographs of synthesized particles collected in the filter.

Figure 5. Size distribution of synthesized particles collected in the filter: $(a)$, $\mathrm{SCL}=100 \% ;(b), \mathrm{SCL}=90 \%$; and $(c), \mathrm{SCL}=70 \%$.

Figure 6. Dependence of the mean diameter of synthesized particles on the shimmer current level in modulated coil current.

Figure 7. Dependence of the standard deviation in the diameter of synthesized particles on the shimmer current level in modulated coil current.

Figure 8. Dependence of the specific surface area of synthesized particles on the shimmer current level in modulated coil current. The specific surface area was measured using BET method. 
Figure 9. XRD spectra of synthesized particles collected in the filter: $(a), \mathrm{SCL}=100 \%$; (b), $\mathrm{SCL}=90 \% ;(c), \mathrm{SCL}=80 \% ;(d), \mathrm{SCL}=70 \%$.

Figure 10. Dependence of the weight fraction of anatase $\mathrm{TiO}_{2}$ of synthesized particles on the shimmer current level in modulated coil current.

Figure 11. Spectroscopic observation system and observation positions.

Figure 12. Emission spectra observed in the reaction chamber installed downstream of the plasma torch during feeding Ti raw powder. The observation positions are: $(a)$, at $200 \mathrm{~mm}$ below the coil end; (b), at $370 \mathrm{~mm}$ below the coil end; and (c), at $460 \mathrm{~mm}$ below the coil end.

Figure 13. Calculated radiation intensity $I_{\text {cal }}(\lambda)$ of TiO spectra. $(a), A^{3} \Phi-X^{3} \Delta$ system; (b), $B^{3} \Pi-X^{3} \Delta$ system; $(c), C^{3} \Delta-X^{3} \Delta$ system; and (d), Total.

Figure 14. Fitting aspect between the measured and calculated radiation intensity of $\mathrm{TiO}$ spectra.

Figure 15. Vibrational and rotational temperatures of $\mathrm{TiO}$ estimated from $\mathrm{TiO}$ emission spectra at different axial positions in the reaction chamber. $(a)$, vibrational temperature; $(b)$, rotational temperature. 


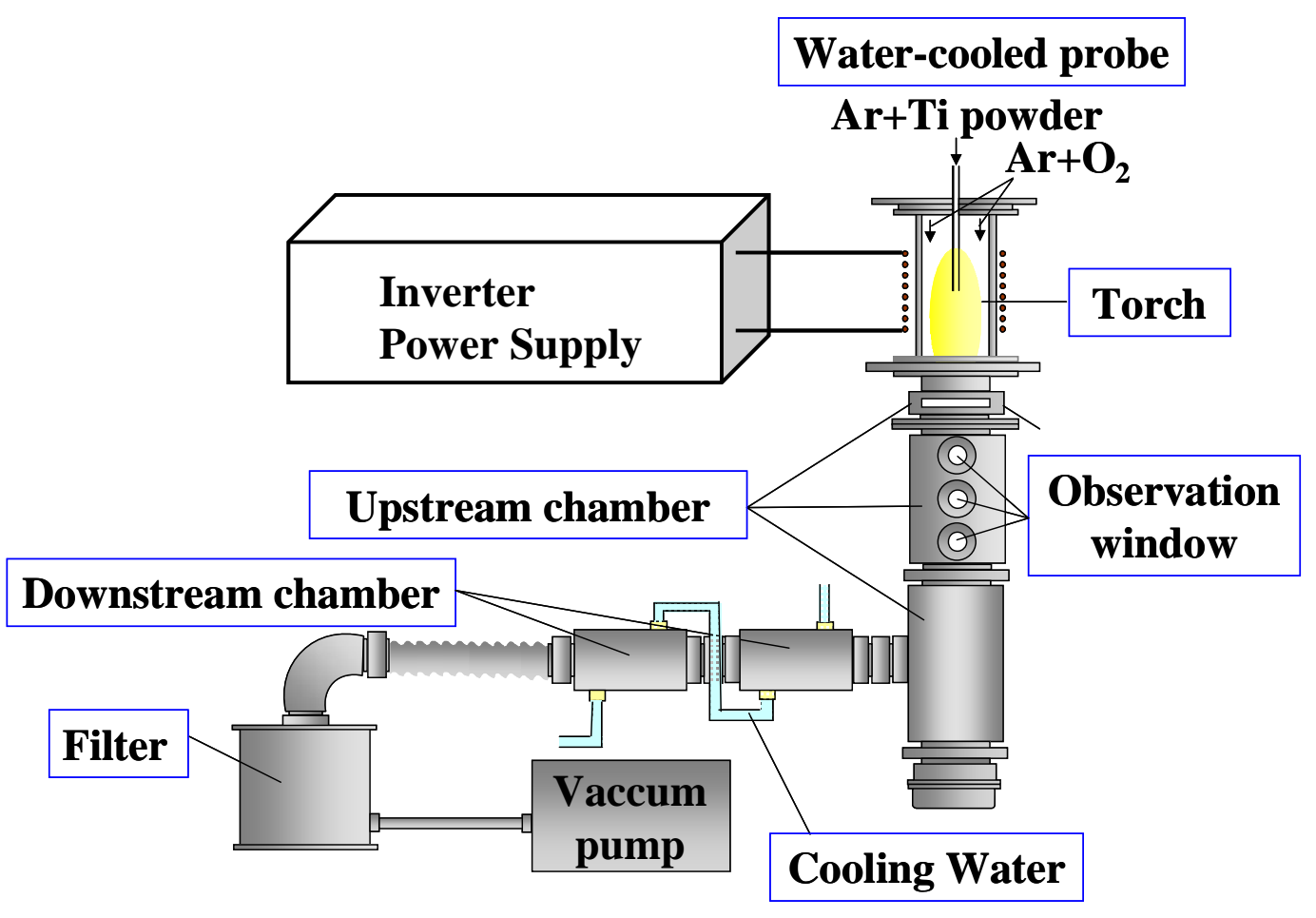

Figure 1. Experimental setup for nanoparticle synthesis. 


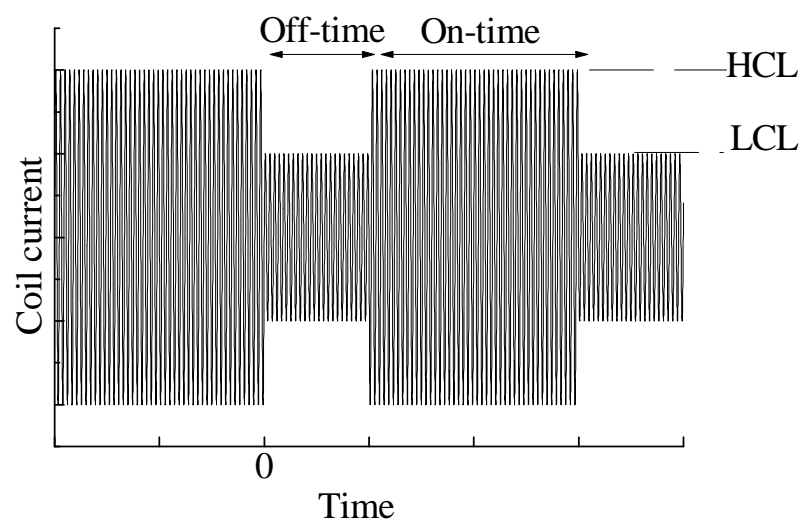

Figure 2. Modulated coil current and definition of modulation parameters. 


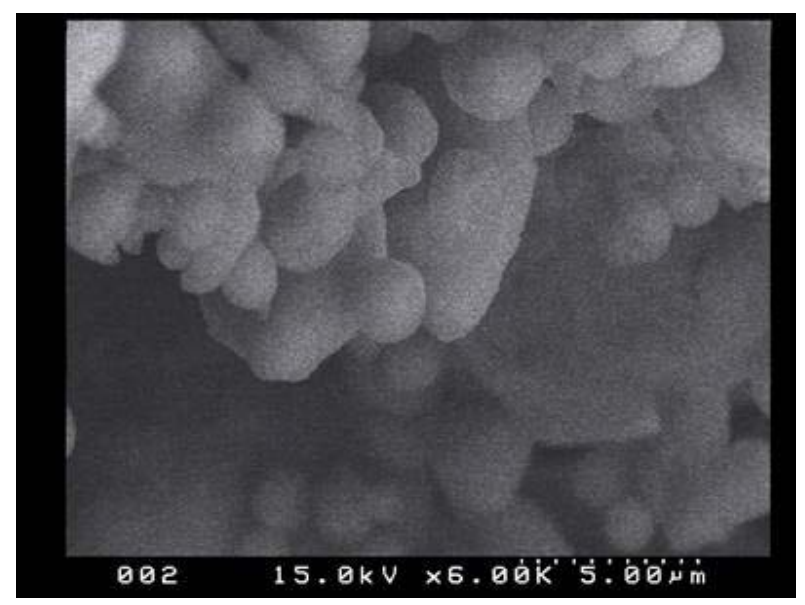

Figure 3. Image of titanium raw powder. 


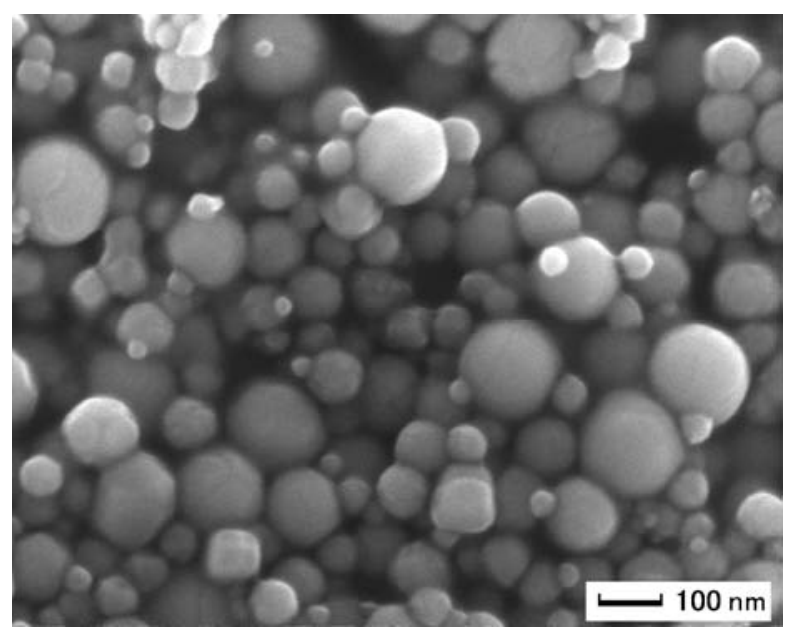

(a) w/o modulation.

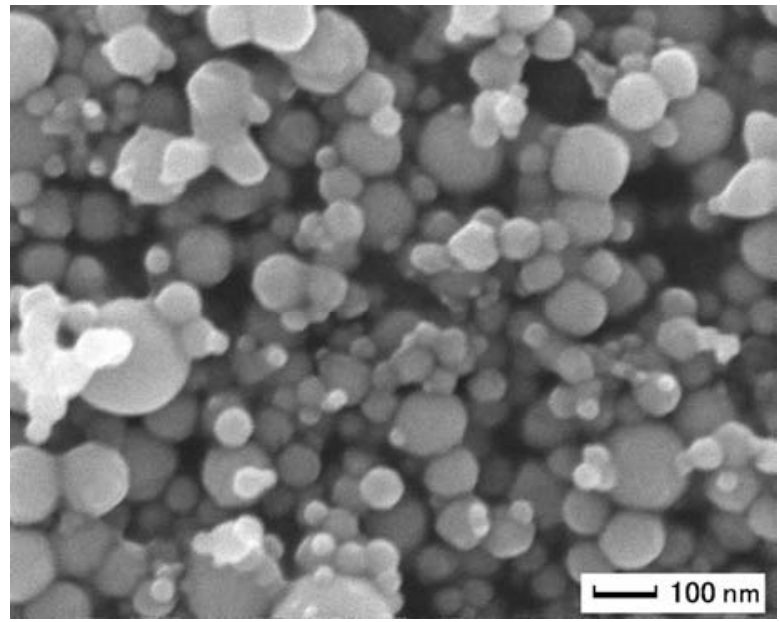

(i) $\mathrm{SCL}=90 \%$.

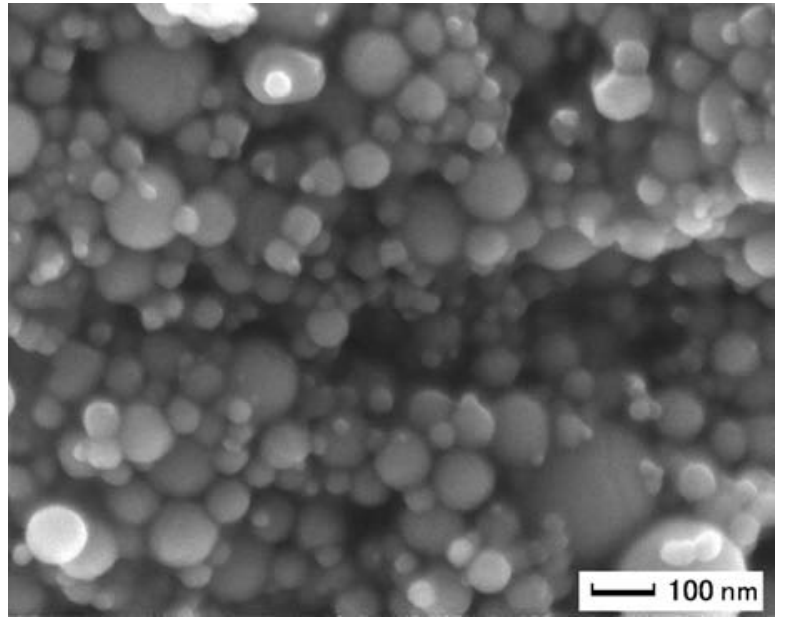

(ii) $\mathrm{SCL}=70 \%$.

(b) with modulation.

Figure 4. SEM micrographs of synthesized particles collected in the filter. 


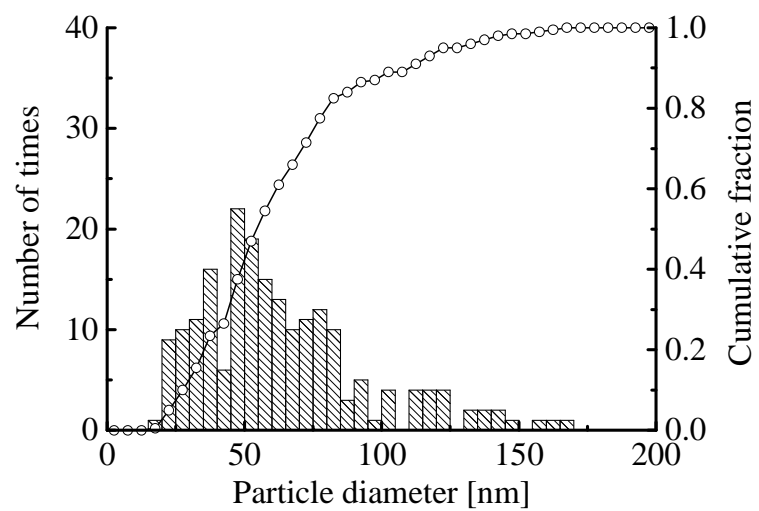

(a) SCL $=100 \%$ (w/o modulation).

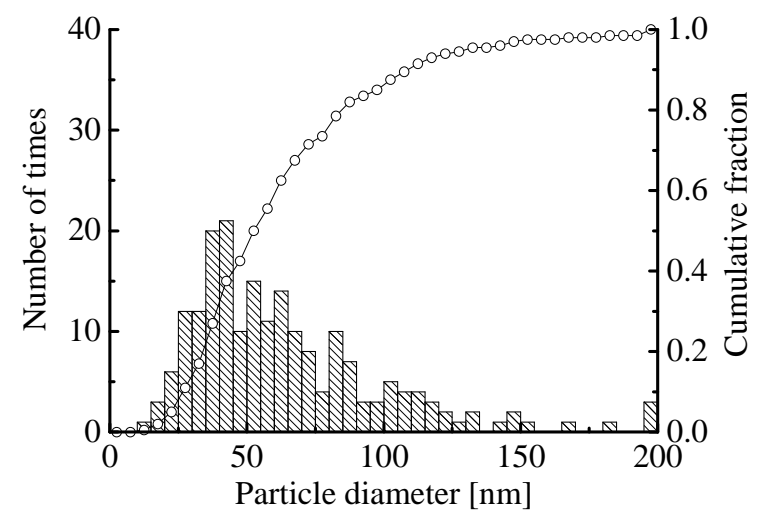

(b) $\mathrm{SCL}=90 \%$.

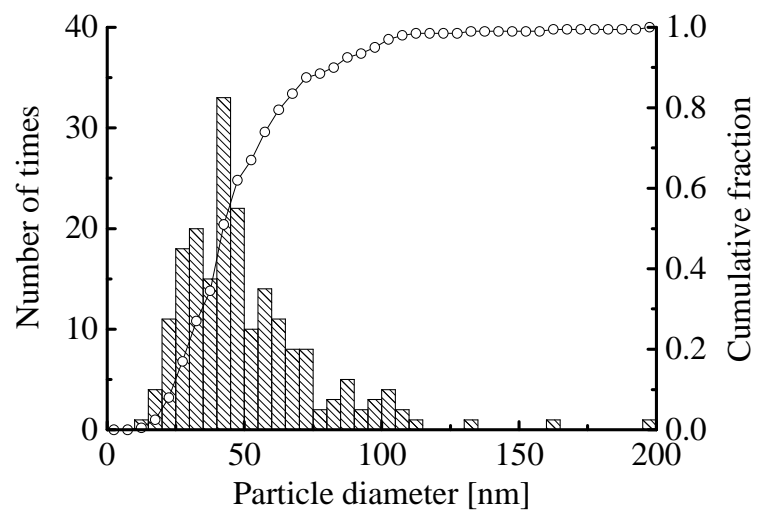

(c) $\mathrm{SCL}=70 \%$.

Figure 5. Size distribution of synthesized particles collected in the filter: $(a)$, $\mathrm{SCL}=100 \%$; $(b), \mathrm{SCL}=90 \%$; and (c), SCL $=70 \%$. 


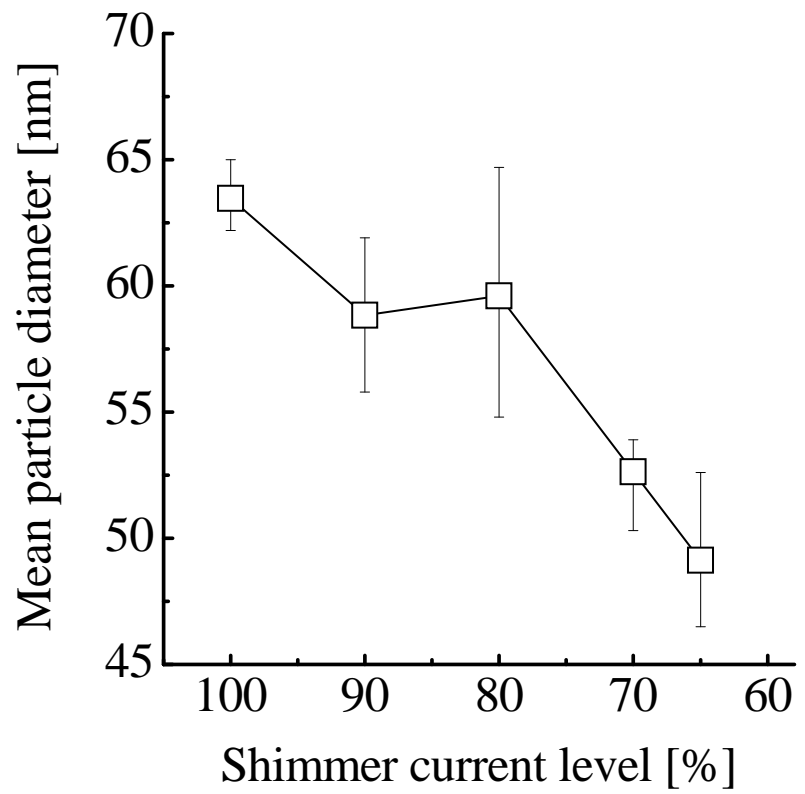

Figure 6. Dependence of the mean diameter of synthesized particles on the shimmer current level in modulated coil current. 


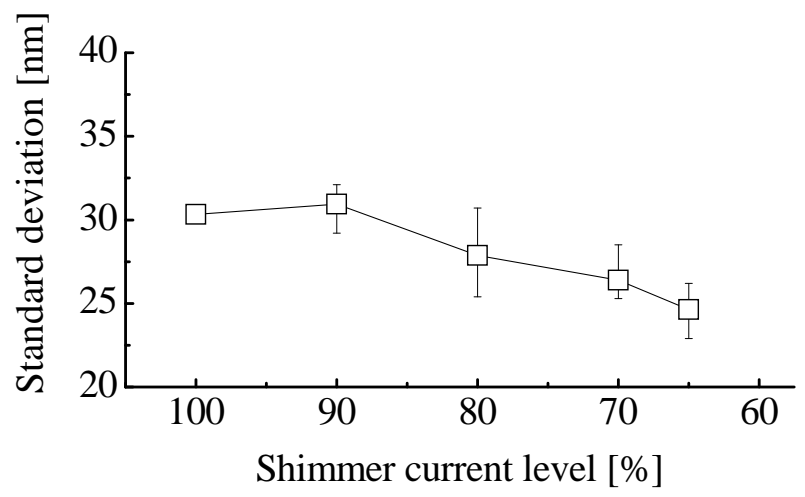

Figure 7. Dependence of the standard deviation in the diameter of synthesized particles on the shimmer current level in modulated coil current. 


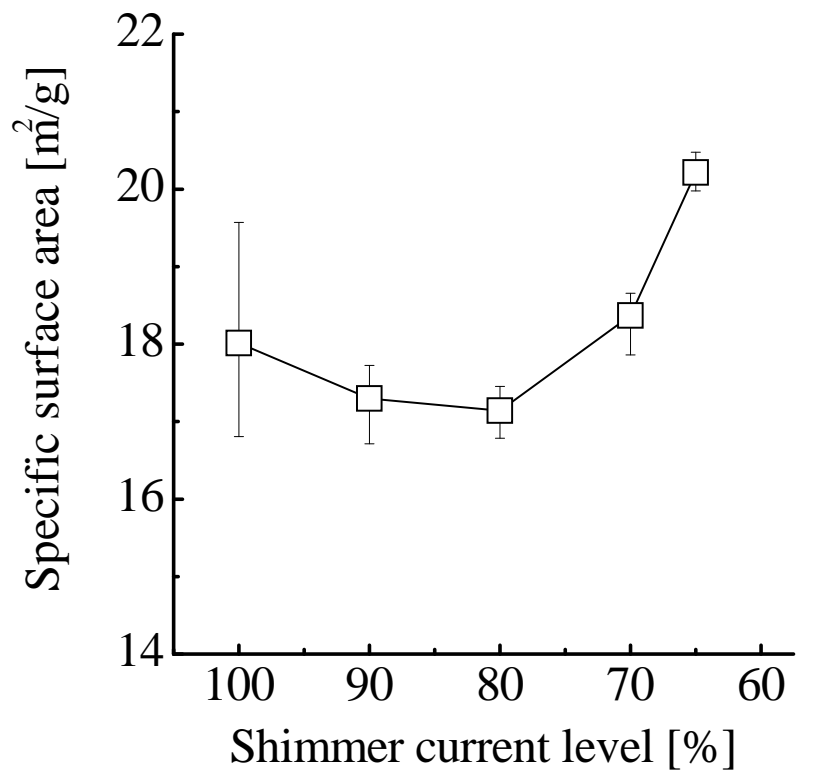

Figure 8. Dependence of the specific surface area of synthesized particles on the shimmer current level in modulated coil current. The specific surface area was measured using BET method. 


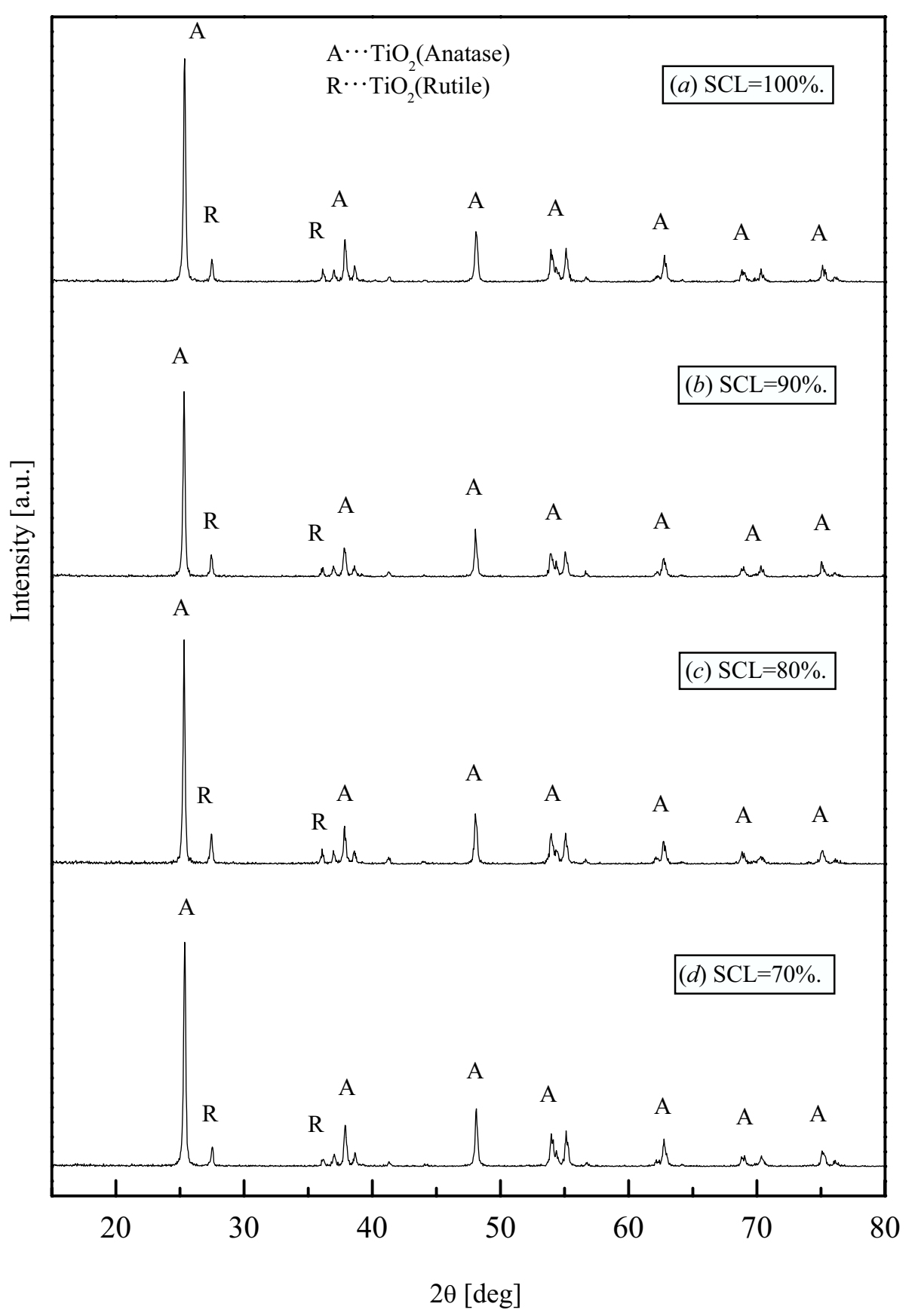

Figure 9. XRD spectra of synthesized particles collected in the filter: $(a), \mathrm{SCL}=100 \%$; (b), $\mathrm{SCL}=90 \% ;(c), \mathrm{SCL}=80 \% ;(d), \mathrm{SCL}=70 \%$. 


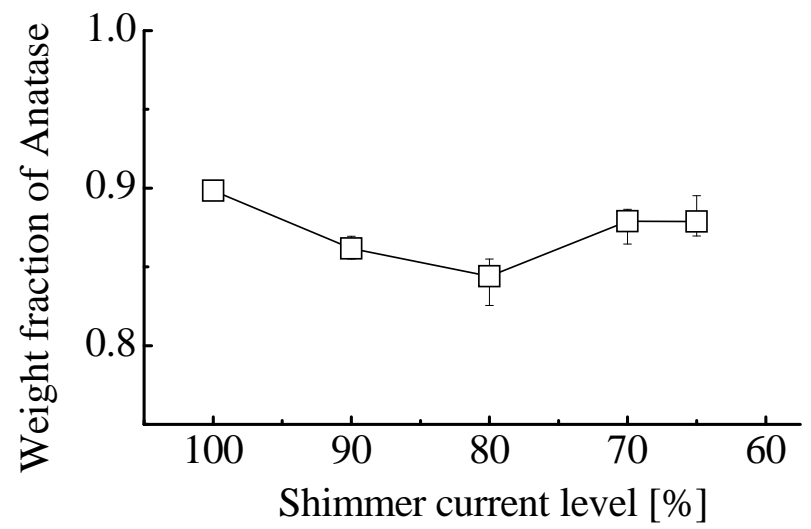

Figure 10. Dependence of the weight fraction of anatase $\mathrm{TiO}_{2}$ of synthesized particles on the shimmer current level in modulated coil current. 


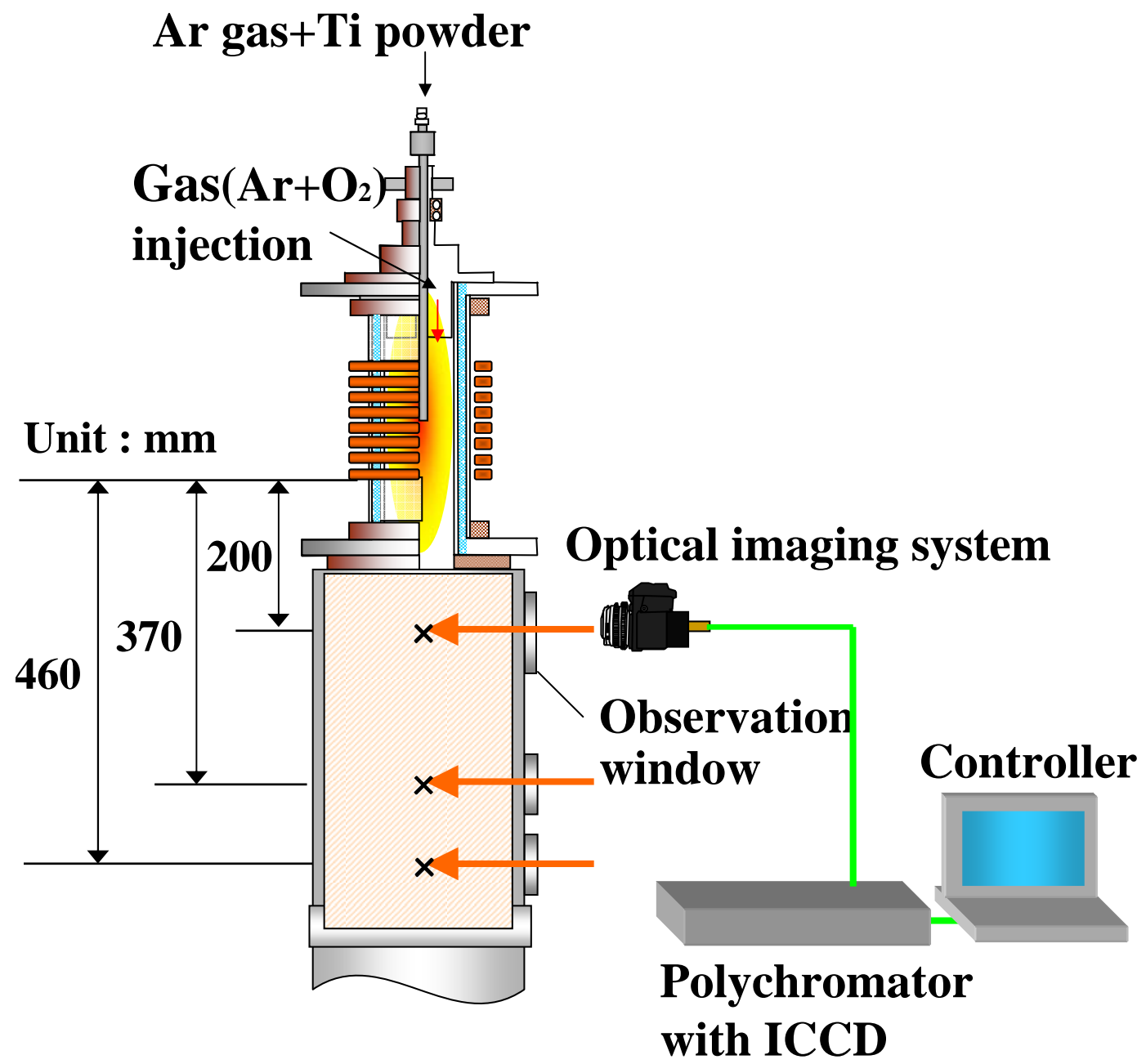

Figure 11. Spectroscopic observation system and observation positions. 


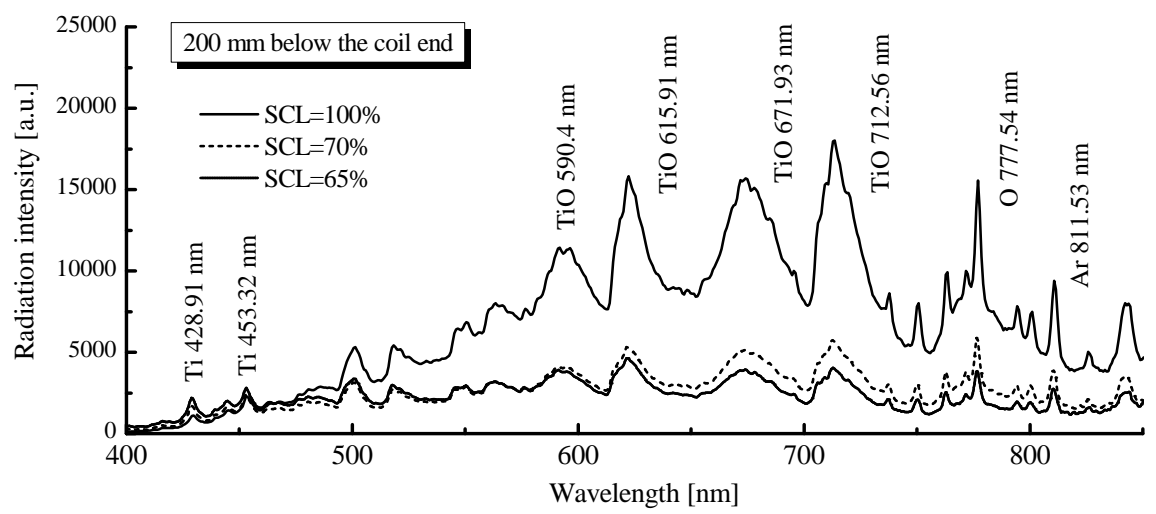

(a) At $200 \mathrm{~mm}$ below the coil end.

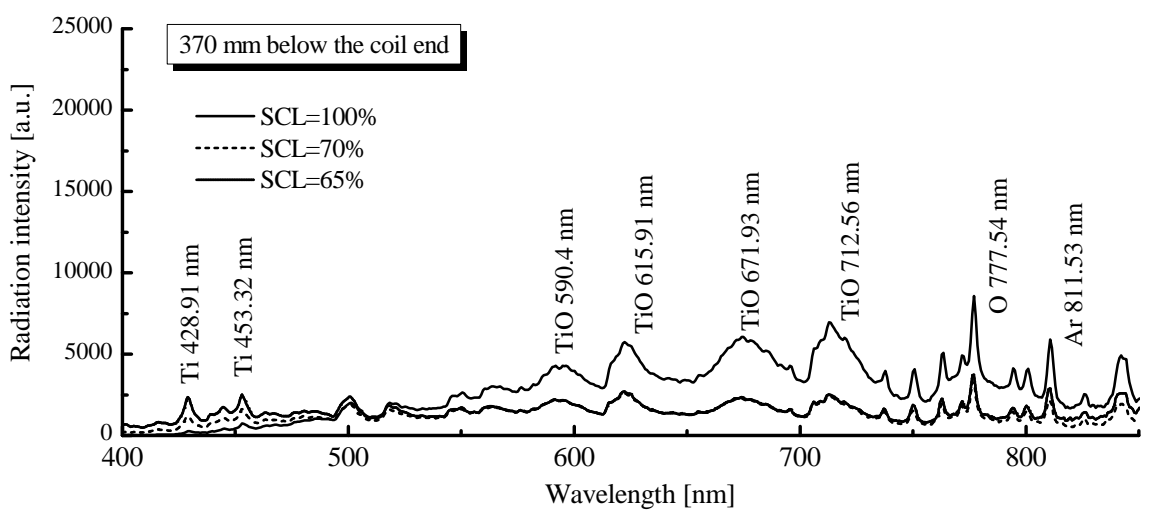

(b) At 370 mm below the coil end.

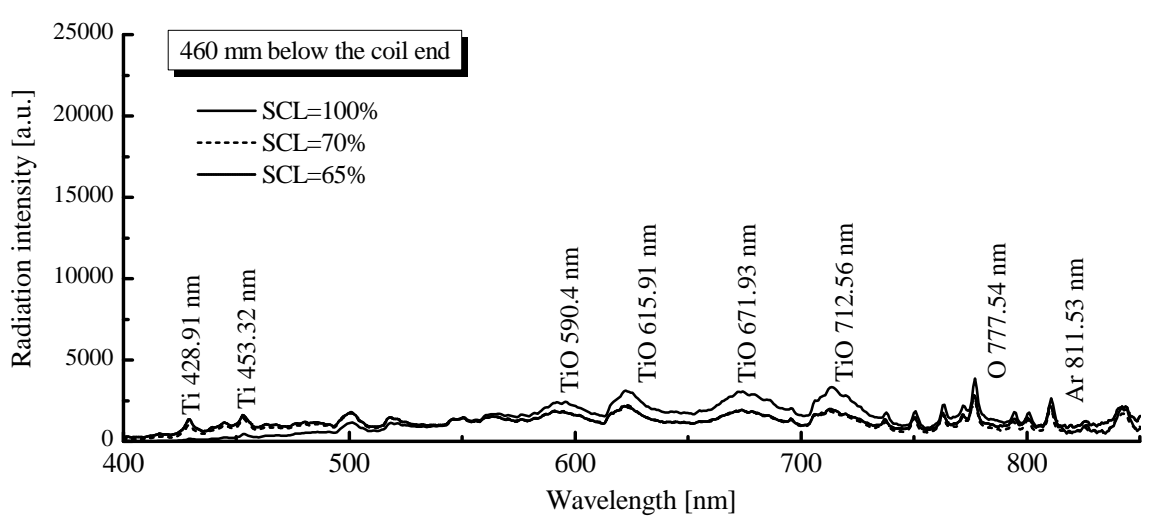

(c) At $460 \mathrm{~mm}$ below the coil end.

Figure 12. Emission spectra observed in the reaction chamber installed downstream of the plasma torch during feeding Ti raw powder. The observation positions are: $(a)$, at $200 \mathrm{~mm}$ below the coil end; (b), at $370 \mathrm{~mm}$ below the coil end; and (c), at $460 \mathrm{~mm}$ below the coil end. 

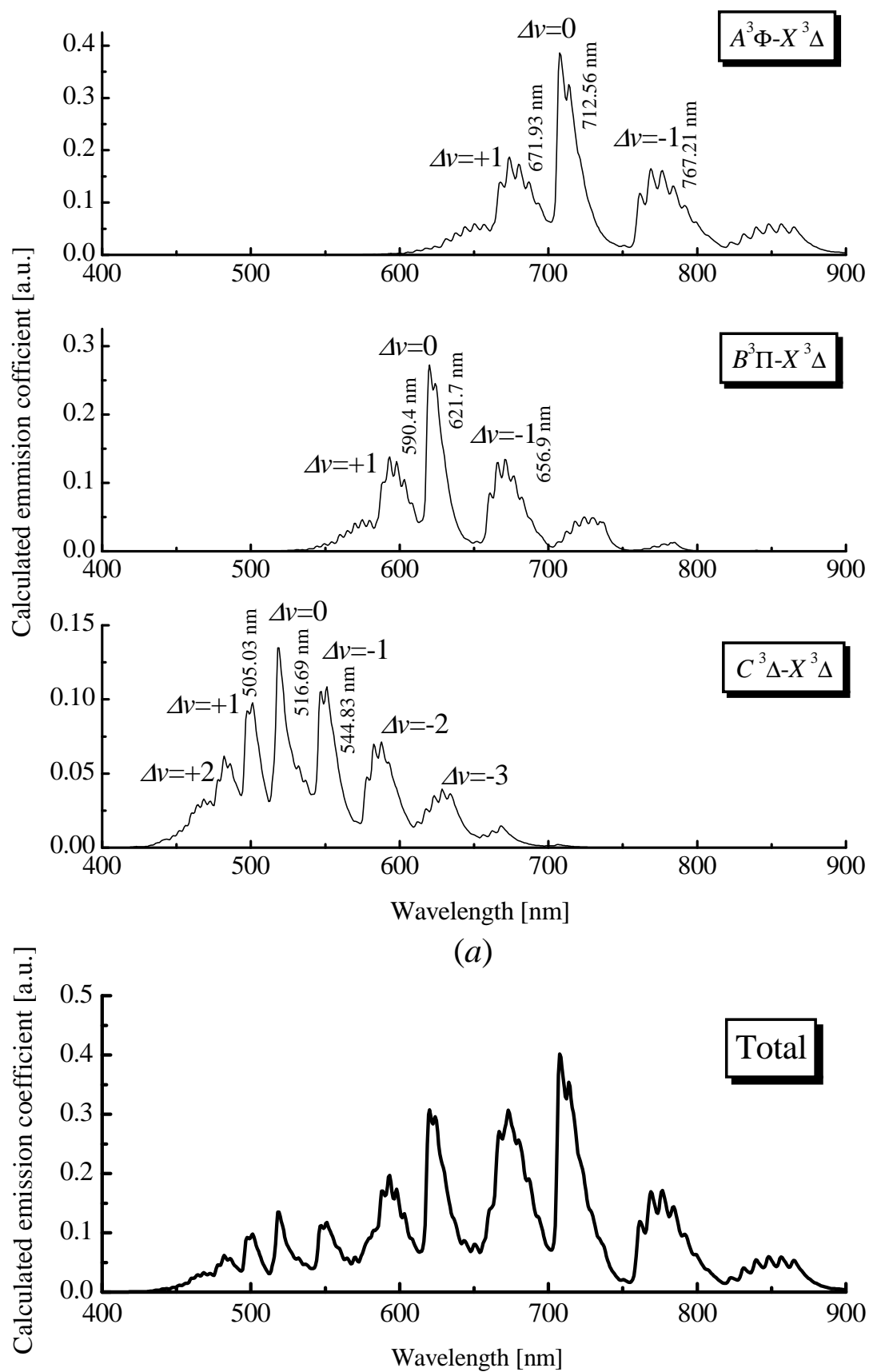

(b)

Figure 13. Calculated radiation intensity $I_{\text {cal }}(\lambda)$ of TiO spectra for vibrational and rotational temperatures of $4000 \mathrm{~K}$. (a), $A^{3} \Phi-X^{3} \Delta$ system; (b), $B^{3} \Pi-X^{3} \Delta$ system; (c), $C^{3} \Delta-X^{3} \Delta$ system; and (d), Total. 


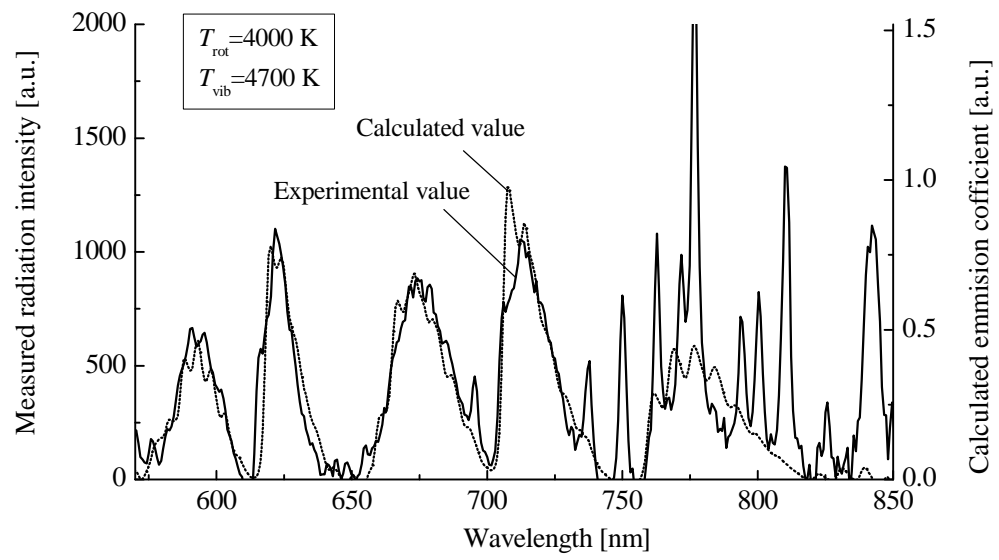

Figure 14. Fitting aspect between the measured and calculated radiation intensity of $\mathrm{TiO}$ spectra. 


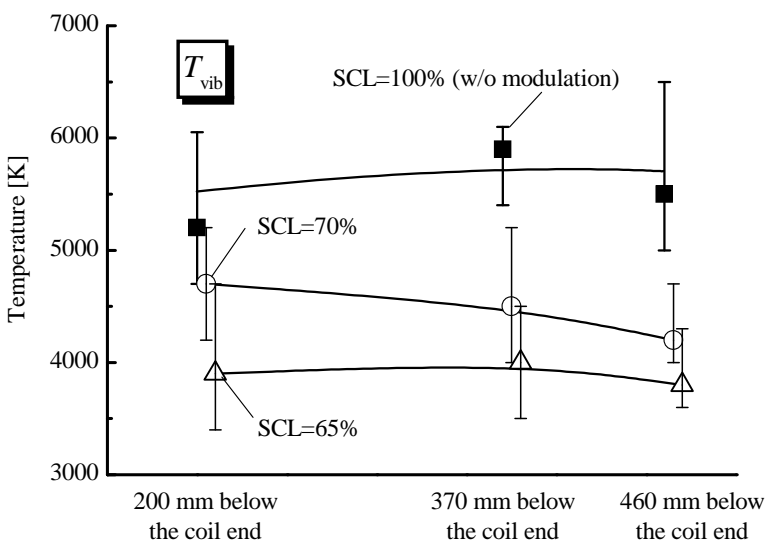

(a)

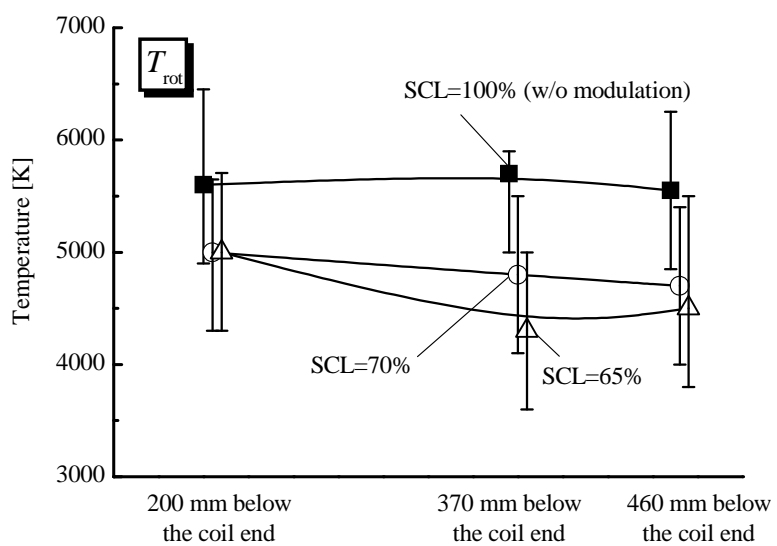

(b)

Figure 15. Vibrational and rotational temperatures of $\mathrm{TiO}$ estimated from $\mathrm{TiO}$ emission spectra at different axial positions in the reaction chamber. $(a)$, vibrational temperature; (b), rotational temperature. 